\title{
A Modified Wallman Method of Compactification
}

\author{
Hueytzen $\mathrm{J}$. $\mathrm{Wu}^{1}$, Wan-Hong $\mathrm{Wu}^{2}$ \\ ${ }^{1}$ Department of Mathematics, Texas A\&M University, Kingsville, USA \\ ${ }^{2}$ University of Texas at San Antonio, San Antonio, USA \\ Email: hueytzen.wu@tamuk.edu, dd1273@yahoo.com
}

Received November 29, 2012; revised December 28, 2012, accepted January 19, 2013

Copyright (C) 2013 Hueytzen J. Wu, Wan-Hong Wu. This is an open access article distributed under the Creative Commons Attribution License, which permits unrestricted use, distribution, and reproduction in any medium, provided the original work is properly cited.

\section{ABSTRACT}

Closed $\wp_{x^{-}}$and basic closed $C^{*}{ }$-filters are used in a process similar to Wallman method for compactifications of the topological spaces $\mathbf{Y}$, of which, there is a subset $D$ of $\mathbf{C} *(\mathbf{Y})$ containing a non-constant function, where $\mathbf{C} *(\mathbf{Y})$ is the set of bounded real continuous functions on $\mathbf{Y}$. An arbitrary Hausdorff compactification $(Z, h)$ of a Tychonoff space $\mathbf{X}$ can be obtained by using basic closed $C^{*}{ }$-filters from $D=\left\{\left.{ }^{\circ} f \circ h\right|^{\circ} f \in{ }^{\circ} D=C(Z)\right\}$ in a similar way, where $C(Z)$ is the set of real continuous functions on $Z$.

Keywords: Closed $\wp_{x}$-Filter; Open and Closed $C^{*}{ }$-Filter Bases; Basic Open and Closed $C^{*}{ }_{D}$-Filters; Compactification; Stone-Čech and Wallman Compactifications

\section{Introduction}

Throughout this paper, $[T]^{<\omega}$ will denote the collection of all finite subsets of the set $T$. For the other notations and the terminologies in general topology which are not explicitly defined in this paper, the readers will be referred to the reference [1].

Let $\mathbf{C} *(\mathbf{Y})$ be the set of bounded real continuous functions on a topological space $\mathbf{Y}$. For any subset $D$ of $\mathbf{C} *(\mathbf{Y})$, we will show in Section 2 that there exists a unique $r_{f}$ in $\operatorname{Cl}(\mathrm{f}(\mathbf{Y}))$ for each $f$ in $D$ so that for any

$$
\begin{aligned}
H & \in[D]^{<\omega}, \varepsilon>0, \phi \neq \cap_{f \in H} f^{-1}\left(\left(r_{f}-\varepsilon, r_{f}+\varepsilon\right)\right) \\
& \subset \cap_{f \in H} f^{-1}\left(\left[r_{f}-\varepsilon, r_{f}+\varepsilon\right]\right) .
\end{aligned}
$$

Let $\mathrm{K}$ be the set

$$
\begin{aligned}
& \left\{\cap_{f \in H} f^{-1}\left(\left[r_{f}-\varepsilon, r_{f}+\varepsilon\right]\right) \mid\right. \\
& \cap_{f \in H} f^{-1}\left(\left[r_{f}-\varepsilon, r_{f}+\varepsilon\right]\right) \neq \phi \\
& \text { for any } \left.H \in[D]^{<\omega}, \varepsilon>0\right\}
\end{aligned}
$$

and let $\mathrm{V}$ be the set

$$
\begin{aligned}
& \left\{\cap_{f \in H} f^{-1}\left(\left(r_{f}-\varepsilon, r_{f}+\varepsilon\right)\right) \mid \cap_{f \in H} f^{-1}\left(\left(r_{f}-\varepsilon, r_{f}+\varepsilon\right)\right) \neq \phi\right. \\
& \text { for any } \left.H \in[D]^{<\omega}, \varepsilon>0\right\}
\end{aligned}
$$

$\mathrm{K}$ and $\mathrm{V}$ are called a closed $C^{*}{ }^{-}$-filter base and an open $C^{*}{ }_{D}$-filter base on $\mathbf{Y}$, respectively. A closed filter (or an open filter) on $\mathbf{Y}$ generated by a $\mathrm{K}$ (or a $\mathrm{V}$ ) is called a basic closed $C^{*}{ }^{-}$filter (or a basic open $C^{*} D^{-}$ filter), denoted by $\mathcal{E}$ (or $\AA$ ). If $r_{f}=f(x)$ for all $f$ in $D$ at some $x$ in $\mathbf{Y}$, then $\mathrm{K}, \mathrm{V}, \mathcal{E}$ and $\AA$ are denoted by $\mathrm{K}_{x}$, $\mathrm{V}_{x}, \mathcal{E}_{x}$ and $\AA_{x}$, respectively. Let $\mathbf{Y}$ be a topological space, of which, there is a subset $D$ of $\mathbf{C} *(\mathbf{Y})$ containing a non-constant function. A compactification $\left(Y^{w}, \mathfrak{I}\right)$ of $\mathbf{Y}$ is obtained by using closed $\wp_{\mathrm{x}^{-}}$and basic closed $C^{*}{ }^{*}$-filters in a process similar to the Wallman method, where $Y^{w}=Y_{E} \cup Y_{F}, Y_{E}$ is the set $\left\{\mathbf{N}_{x} \mid \mathbf{N}_{x}\right.$ is a closed $\wp_{x}$-filter, $x$ is in $\left.\mathbf{Y}\right\}, Y_{F}$ is the set of all basic closed $C^{*}{ }^{*}$-filter that does not converge in $\mathbf{Y}, \mathfrak{I}$ is the topology induced by the base $\tau=\left\{F^{*} \mid F\right.$ is a nonempty closed set in $\mathbf{Y}$ \} for the closed sets of $Y^{w}$ and $F^{*}$ is the set of all $\mathfrak{C}$ in $Y^{w}$ such that $F \cap T \neq \phi$ for all $T$ in $\mathfrak{C}$. Similarly, an arbitrary Hausdorff compactification $(\mathbf{Z}, \mathrm{h})$ of a Tychonoff space $\mathbf{X}$ can be obtained by using the basic closed $C^{*} D^{- \text {filters }}$ on $\mathbf{X}$ from $D=\left\{\left.{ }^{\circ} f \circ h\right|^{\circ} f \in{ }^{\circ} D\right\}$, where ${ }^{\circ} D$ is the set $\mathbf{C}^{*}(\mathbf{z})$.

\section{Open and Closed $C^{*}{ }^{*}$-Filter Bases, Basic Open and Closed $C^{*} D^{*}$ Filters}

For an arbitrary topological space $\mathbf{Y}$, let $D$ be a subset 
of $\mathbf{C} *(\mathbf{Y})$.

Theorem 2.1 Let $\mathcal{F}$ be a filter on $\mathbf{Y}$. For each $f$ in $D$ there exists a $r_{f}$ in $\mathrm{Cl}(\mathrm{f}(\mathbf{Y}))$ such that

$$
f^{-1}\left(\left(r_{f}-\varepsilon, r_{f}+\varepsilon\right)\right) \cap F \neq \phi
$$

for any $F$ in $\mathcal{F}$ and any $\varepsilon>0$ (See Thm. 2.1 in $[2$, p.1164]).

Proof. If the conclusion is not true, then there is an $f$ in $D$ such that for each $r_{t}$ in $\mathrm{Cl}(\mathrm{f}(\mathbf{Y}))$ there exist an $F_{t}$ in $\mathcal{F}$ and an $\varepsilon_{t}>0$ such that

$$
F_{t} \cap f^{-1}\left(\left(r_{t}-\varepsilon_{t}, r_{t}+\varepsilon_{t}\right)\right)=\phi .
$$

Since $\mathrm{Cl}(\mathrm{f}(\mathbf{Y}))$ is compact and $\mathrm{Cl}(\mathrm{f}(\mathbf{Y}))$ is contained in

$$
\cup\left\{\left(r_{t}-\varepsilon_{t}, r_{t}+\varepsilon_{t}\right) \mid r_{t} \text { is in } \mathrm{Cl}(\mathrm{f}(\mathbf{Y}))\right\},
$$

there exist $r_{1}, \cdots, r_{n}$ in $\mathrm{Cl}(\mathrm{f}(\mathbf{Y}))$ such that $\mathbf{Y}$ is contained in

$$
\cup\left\{f^{-1}\left(\left(r_{i}-\varepsilon_{i}, r_{i}+\varepsilon_{i}\right)\right) \mid i=1, \cdots, n\right\} .
$$

Let $F_{\circ}=\cap\left\{F_{i} \mid i=1, \cdots, n\right\}$, then $F_{\circ}$ is in $\mathcal{F}$ and

$$
F_{\circ} \subseteq \cup\left\{F_{i} \cap f^{-1}\left(\left(r_{i}-\varepsilon_{i}, r_{i}+\varepsilon_{i}\right)\right) \mid i=1, \cdots, n\right\}=\phi,
$$

contradicting that $\phi$ is not in $\mathcal{F}$.

Corollary 2.2 Let $\mathcal{F}$ (or $\mathrm{Q}$ ) be a closed (or an open) ultrafilter on $\mathbf{Y}$. For each $f$ in $D$, there exists a unique $r_{f}$ in $\mathrm{Cl}(\mathrm{f}(\mathbf{Y}))$ such that (1) for any $H \in[D]^{<\omega}$, any $\varepsilon>0$,

$$
\begin{aligned}
& \cap_{f \in H} f^{-1}\left(\left[r_{f}-\varepsilon, r_{f}+\varepsilon\right]\right) \in \mathcal{F} \\
& \left(\text { or } \cap_{f \in H} f^{-1}\left(\left(r_{f}-\varepsilon, r_{f}+\varepsilon\right)\right) \in \mathrm{Q}\right)
\end{aligned}
$$

and (2) for any $H \in[D]^{<\omega}$, any $\varepsilon>0$,

$$
\begin{aligned}
& \cap_{f \in H} f^{-1}\left(\left[r_{f}-\varepsilon, r_{f}+\varepsilon\right]\right) \neq \phi \\
& \left(\text { or } \cap_{f \in H} f^{-1}\left(\left(r_{f}-\varepsilon, r_{f}+\varepsilon\right)\right) \neq \phi\right) .
\end{aligned}
$$

(See Cor. 2.2 \& 2.3 in [2, p.1164].)

Therefore, for a given closed ultrafilter $\mathcal{F}$ (or open ultrafilter Q), there exists a unique $r_{f}$ in $\mathrm{Cl}(\mathrm{f}(\mathbf{Y}))$ for each $f$ in $D$ such that for any $H \in[D]^{<\omega}, \varepsilon>0$,

$$
\begin{aligned}
& \cap_{f \in H} f^{-1}\left(\left[r_{f}-\varepsilon, r_{f}+\varepsilon\right]\right) \neq \phi \\
& \left(\text { or } \cap_{f \in H} f^{-1}\left(\left(r_{f}-\varepsilon, r_{f}+\varepsilon\right)\right) \neq \phi\right) .
\end{aligned}
$$

Let $\mathrm{K}$ be the set

$$
\begin{aligned}
& \left\{\cap_{f \in H} f^{-1}\left(\left[r_{f}-\varepsilon, r_{f}+\varepsilon\right]\right) \mid\right. \\
& \cap_{f \in H} f^{-1}\left(\left[r_{f}-\varepsilon, r_{f}+\varepsilon\right]\right) \neq \phi \\
& \text { for any } \left.H \in[D]^{<\omega}, \varepsilon>0\right\}
\end{aligned}
$$

and let $\mathrm{V}$ be the set

$$
\begin{aligned}
& \left\{\cap_{f \in H} f^{-1}\left(\left(r_{f}-\varepsilon, r_{f}+\varepsilon\right)\right) \mid\right. \\
& \cap_{f \in H} f^{-1}\left(\left(r_{f}-\varepsilon, r_{f}+\varepsilon\right)\right) \\
& \left.\neq \phi, \text { for any } H \in[D]^{<\omega}, \varepsilon>0\right\}
\end{aligned}
$$

$\mathrm{K}$ and $\mathrm{V}$ are called a closed and an open $C^{*}{ }^{\text {-filter }}$ bases, respectively. If for all $f$ in $D, \mathrm{r}_{\mathrm{f}}=\mathrm{f}(\mathrm{x})$ for some $x$ in $Y$, then $K$ and $V$ are called the closed and open $C^{*}{ }^{-}$-filter bases at $\boldsymbol{x}$, denoted by $\mathrm{K}_{x}$ and $\mathrm{V}_{x}$, respectively. Let $\mathcal{E}$ and $\mathcal{E}_{x}$ (or $\AA$ and $\AA_{x}$ ) be the closed (or open) filters generated by $\mathrm{K}$ and $\mathrm{K}_{x}$ (or $\mathrm{V}$ and $\mathrm{V}_{x}$ ), respectively, then $\mathcal{E}$ and $\mathcal{E}_{x}$ (or $\AA$ and $\AA_{x}$ ) are called a basic closed $C^{*}{ }^{*}$-filter and the basic closed $C^{*}{ }$-filter at $x$ (or a basic open $C^{*}{ }$-filter and the basic open $C^{*}{ }^{*}$-filter at $x$ ), respectively.

Corollary 2.3 Let $\mathcal{F}$ and $\mathrm{Q}$ be a closed and an open ultrafilters on a topological space $\mathbf{Y}$, respectively. Then there exist a unique basic closed $\boldsymbol{C}^{*}{ }^{*}$-filter $\mathcal{E}$ and $a$ unique basic open $\boldsymbol{C}^{*}{ }^{-}$-filter $\AA$ on $\mathbf{Y}$ such that $\mathcal{E}$ is contained in $\mathcal{F}$ and $\AA$ is contained in $\mathrm{Q}$.

\section{A Closed $\wp_{x}$-Filter and a Modified Wallman Method of Compactification}

Let $\mathbf{Y}$ be a topological space, of which, there is a subset $D$ of $\mathbf{C} *(\mathbf{Y})$ containing a non-constant function. For each $\mathbf{x}$ in $\mathbf{Y}$, let $\mathbf{N}_{x}$ be the union of $\{\{\mathrm{x}\}\}$ and $\boldsymbol{\varepsilon}_{x}$, if $\mathbf{V}_{x}$ is an open nhood filter base at $x$; let $\mathrm{N}_{\mathrm{x}}$ be the union of $\{\{\mathrm{x}\}\}$ and $\{F \mid F$ is closed, $x$ is in $F\}$, if $\mathrm{V}_{x}$ is not an open nhood filter base at $x$. For each $x$ in $\mathbf{Y}, \mathbf{N}_{x}$ is a $\wp$-filter with $\wp$ being $\mathbf{N}_{x}$. (See 12E. in [1, p.82] for definition and convergence). $\mathbf{N}_{x}$ is called a closed $\wp_{x}$-filter. It is clear that $\mathrm{K}_{x}$ is contained in $\boldsymbol{E}_{x}$ and $\boldsymbol{E}_{x}$ is contained in $\mathbf{N}_{x}, \mathbf{N}_{x}$ converges to $\mathbf{x}$ for each $x$ in $\mathbf{Y}$. Let $Y_{E}$ be the set of all $\mathbf{N}_{x}, x$ in $\mathbf{Y}$. Let $Y_{F}$ be the set of all basic closed $C^{*}{ }^{*}$-filter $\mathcal{E}$ that does not converge in $\mathbf{Y}$ and let $Y^{w}=Y_{E} \cup Y_{F}$.

Definition 3.4 For each nonempty closed set $\mathbf{F}$ in $\mathbf{Y}$, let $\mathbf{F}^{*}$ be the set of $\mathfrak{C}$ in $Y^{w}$ such that the intersection of $\boldsymbol{F}$ and $\mathbf{T}$ is not an empty set for all $\mathbf{T}$ in $\mathfrak{C}$.

From the Def. 3.4, the following Cor. 3.5 can be readily proved. We omit its proofs.

Corollary 3.5 For a closed set $\mathbf{F}$ in $\mathbf{Y}$, (i) $x$ is in $\mathbf{F}$ if $\mathbf{N}_{\mathbf{x}}$ is in $\mathbf{F}^{*}$; (ii) $\mathbf{F}$ is equal to $\mathbf{Y}$ if $\mathbf{F}^{*}$ is equal to $Y^{w}$; (iii) if $\mathbf{F}$ is in $\mathfrak{C}$, then $\mathfrak{C}$ is in $\mathbf{F}^{*}$; (iv) $\mathfrak{C}$ is in $\left(Y^{w}-\mathbf{F}^{*}\right)$ if there is a $\mathbf{T}$ in $\mathfrak{C}$ such that $\mathbf{T}$ is contained in $\mathbf{Y}-\mathbf{F}$.

Lemma 3.6 For any two nonempty closed sets $E$ and $F$ in $\mathbf{Y}$,

(i) $E \subseteq F \Leftrightarrow E^{*} \subseteq F^{*}$,

(ii) $(E \cap F)^{*} \subseteq\left(E^{*} \cap F^{*}\right)$,

(iii) $(E \cup F)^{*}=\left(E^{*} \cup F^{*}\right)$.

Proof. (i) For [ $\Leftarrow$ ]: If $E \not \subset F$, pick an $\mathrm{x}$ in $E-F$, by 
Cor. 3.5 (i), $\mathrm{N}_{\mathrm{x}}$ is in $E^{*}$ and $\mathrm{N}_{\mathrm{x}}$ is not in $F^{*}$; i.e., $E^{*} \not \subset F^{*}$. For $(\Rightarrow)$ is obvious. (ii) is clear from (i). (iii) For $[\subseteq]$ : If $\mathfrak{C}$ belongs to $(E \cup F)^{*}$ and does not belong $E^{*} \cup F^{*}$, then pick $T_{1}, T_{2}$ in $\mathfrak{C}$ such that

$$
E \cap T_{1}=F \cap T_{2}=\phi .
$$

Since $T_{1} \cap T_{2}$ is in $\mathfrak{C}$ and

$$
(E \cup F) \cap\left(T_{1} \cap T_{2}\right) \subset\left(E \cap T_{1}\right) \cup\left(F \cap T_{2}\right)=\phi .
$$

Thus, $\mathfrak{C}$ does not belong to $(E \cup F)^{*}$, contradicting the assumption. For [つ] is obvious from (i).

Proposition 3.7 $\tau=\left\{F^{*} \mid F\right.$ is a nonempty closed set in $\mathbf{Y}\}$ is a base for the closed sets of $Y^{w}$.

Proof. Let $\mathcal{B}$ be the set $\left\{Y^{w}-F^{*} \mid F^{*} \in \tau\right\}$. We show that $\mathcal{B}$ is a base for $Y^{w}$. For (a) of Thm. 5.3 in [1, p.38], if $\mathfrak{C} \in Y^{w}$, then there exist an $f$ in $D$, a $\delta>0$ such that

$$
S=f^{-1}\left(\left[r_{f}-\delta, r_{f}+\delta\right]\right) \in \mathrm{K} \subset \mathcal{E} \subseteq \mathfrak{C}
$$

and

$$
E=\mathbf{Y}-f^{-1}\left(\left[r_{f}-2 \delta, r_{f}+2 \delta\right]\right) \neq \phi,
$$

otherwise, if for all $f$ in $D$, all $\delta>0, E=\phi$, then for all $f$ in $D, \mathrm{f}(\mathbf{Y})=\left\{r_{f}\right\}$, contradicting that $D$ contains a non-constant function. Thus $E \neq \phi, E$ is closed, $S$ is in $\mathfrak{C}$ and $S \cap E=\phi$ imply that $\mathfrak{C}$ is in $Y^{w}-E^{*}$. So,

$$
X^{w}=\cup\left\{\left(Y^{w}-E^{*}\right) \mid E^{*} \in \tau\right\} .
$$

For (b) of Thm. 5.3, if $\mathfrak{C}$ belongs to

$$
\left(Y^{w}-E^{*}\right) \cap\left(Y^{w}-F^{*}\right),
$$

then $E \cup F$ is closed, $(E \cup F)^{*} \in \tau$ and

$$
\left(Y^{w}-E^{*}\right) \cap\left(Y^{w}-F^{*}\right)=Y^{w}-(E \cup F)^{*}
$$

is in $\mathcal{B}$. Thus, $\mathfrak{C}$ is in

$$
Y^{w}-(E \cup F)^{*} \subseteq\left(Y^{w}-E^{*}\right) \cap\left(Y^{w}-F^{*}\right) .
$$

Equip $Y^{w}$ with the topology $\mathfrak{I}$ induced by $\tau$. For each $f \in D$, define $\mathrm{f}^{*}: Y^{w} \rightarrow \mathbf{R}$ by $f^{*}(\mathfrak{C})=r_{f}$, if

$$
f^{-1}\left(\left[r_{f}-\varepsilon, r_{f}+\varepsilon\right]\right) \in \mathrm{K} \subset \mathcal{E} \subseteq \mathfrak{C}
$$

for all $\varepsilon>0$. Since (i) if $\mathfrak{C}$ is equal to $\mathrm{N}_{x}$ for some $\mathrm{N}_{x}$ in $Y_{E}$, then

$$
f^{-1}([f(x)-\varepsilon, f(x)+\varepsilon])
$$

is in $\mathrm{N}_{x}$ for all $\varepsilon>0$, (ii) if $\mathfrak{C}$ is $\mathcal{E}$ which is in $Y_{F}$, then

$$
f^{-1}\left(\left[r_{f}-\varepsilon, r_{f}+\varepsilon\right]\right)
$$

is in $\mathcal{E}$ for all $\varepsilon>0$, (iii) by Cor. 2.2, the $r_{f}$ is unique for each $f$ in $D$ and (iv) the $\mathrm{K}$ that is contained in $\mathfrak{C}$ is unique. Thus, $f^{*}$ is well-defined for each $f$ in $D$. For all $f$ in $D$, all $x$ in $\mathbf{Y}$,

$$
f^{-1}([f(x)-\varepsilon, f(x)+\varepsilon])
$$

is in $\mathbf{N}_{x}$ for all $\varepsilon>0$, thus $f^{*}\left(\mathbf{N}_{x}\right)$ is equal to $f(x)$ for all $f$ in $D$ and all $x$ in $\mathbf{Y}$.

Lemma 3.8 For each $f$ in $D$, let $r$ be in $C l(f(\mathbf{Y}))$, then

(i) $\left(f^{-1}([r-\delta, r+\delta])\right) * \subseteq f^{*-1}((r-\varepsilon, r+\varepsilon))$ and

$($ ii $) f^{*-1}((r-\varepsilon, r+\varepsilon)) \subseteq\left(f^{-1}([r-\varepsilon, r+\varepsilon])\right) *$

for any $\varepsilon>\delta>0$.

Proof. (i): If $\mathfrak{C}$ is in $\left(f^{-1}([r-\delta, r+\delta])\right) *$ and $f^{*}(\mathfrak{C})$ is $t_{f}$, then

$$
f^{-1}([r-\delta, r+\delta]) \cap f^{-1}\left(\left[t_{f}-\gamma, t_{f}+\gamma\right]\right) \neq \phi
$$

for all $\gamma>0$, where $f^{-1}\left(\left[t_{f}-\gamma, t_{f}+\gamma\right]\right) \in \mathrm{K} \subset \mathfrak{C}$ for all $\gamma>0$. Thus,

$$
[r-\delta, r+\delta] \cap\left[t_{f}-\gamma, t_{f}+\gamma\right] \neq \phi
$$

for all $\gamma>0$; i.e., $\mathrm{f} *(\mathfrak{C})$ is

$$
t_{f} \in[r-\delta, r+\delta] \subseteq(r-\varepsilon, r+\varepsilon),
$$

so $\mathfrak{C}$ is in $f^{*^{-1}}((r-\varepsilon, r+\varepsilon))$. For (ii): If $\mathfrak{C}$ is in $f^{*-1}((r-\varepsilon, r+\varepsilon))$ and $\mathrm{f} *(\mathfrak{C})$ is $t_{f}$, then

$$
t_{f} \in(r-\varepsilon, r+\varepsilon) \text {. }
$$

Pick a $\delta>0$ such that

$$
\left[t_{f}-\delta, t_{f}+\delta\right] \subset[r-\varepsilon, r+\varepsilon],
$$

then

$$
f^{-1}\left(\left[t_{f}-\delta, t_{f}+\delta\right]\right) \subset f^{-1}([r-\varepsilon, r+\varepsilon]) .
$$

Since

$$
f^{-1}\left(\left[t_{f}-\delta, t_{f}+\delta\right]\right) \in \mathrm{K} \subset \mathfrak{C},
$$

thus $f^{-1}([r-\varepsilon, r+\varepsilon]) \in \mathfrak{C}$. By Cor. 3.5 (iii), $\mathfrak{C}$ is in $\left(f^{-1}([r-\varepsilon, r+\varepsilon])\right)^{*}$.

Proposition 3.9 For each $f$ in $D, f^{*}$ is a bounded real continuous function on $Y^{w}$.

Proof. For each $f$ in $D$ and each $\mathfrak{C}$ in $Y^{w}, \mathrm{f}^{*}(\mathfrak{C})$ is in $\mathrm{Cl}(\mathrm{f}(\mathbf{Y}))$. Thus $f^{*}\left(Y^{w}\right)$ is contained in $\mathrm{Cl}(\mathrm{f}(\mathbf{Y}))$; i.e., $f^{*}$ is bounded on $Y^{w}$. For the continuity of $f^{*}$ : If $\mathfrak{C}$ is in $Y^{w}$ and $f^{*}(\mathfrak{C})$ is $t_{f}$. We show that for any $\varepsilon>0$, there is a $E^{*}$ in $\tau$ such that $\mathfrak{C}$ is in

$$
U=Y^{w}-E^{*} \subset f^{*-1}\left(\left(t_{f}-\varepsilon, t_{f}+\varepsilon\right)\right) .
$$

Let 


$$
E=f^{-1}\left(\left(-\infty, t_{f}-\varepsilon / 2\right]\right) \cup f^{-1}\left(\left[t_{f}+\varepsilon / 2, \infty\right)\right)
$$

and $U=Y^{w}-E^{*}$. Since

$$
P=f^{-1}\left(\left[t_{f}-\varepsilon / 3, t_{f}+\varepsilon / 3\right]\right) \in \mathrm{K} \subset \mathfrak{C}
$$

and $P \subset Y-E$, by Cor. 3.5 (iv), $\mathfrak{C} \in U$. Next, for any $\mathfrak{C}_{s}$ in $U$, if $\mathfrak{C}_{s} \neq \mathbf{N}_{x}$ for all $x$ in $\mathbf{Y}$, by Cor. $\mathbf{3 . 5}$ (iv), pick a $T$ in $\mathfrak{C}_{s}$ such that

$$
T \subset Y-E \subset f^{-1}\left(\left[t_{f}-\varepsilon / 2, t_{f}+\varepsilon / 2\right]\right)=S,
$$

then $S$ is in $\mathfrak{C}_{s}$. By Cor. 3.5 (iii) and Lemma 3.8 (i), $\mathfrak{C}_{s}$ is in $S^{*} \subset f^{*-1}\left(\left(t_{f}-\varepsilon, t_{f}+\varepsilon\right)\right)$. If $\mathfrak{C}_{s}$ is $\mathbf{N}_{x}$ for some $x$ in $\mathbf{Y}$, by Cor. 3.5 (i), $\mathrm{N}_{x}$ in $U$ if $x \notin E$, thus

$$
f^{*}\left(\mathrm{~N}_{x}\right)=f(x) \in\left(t_{f}-\varepsilon / 2, t_{f}+\varepsilon / 2\right) ;
$$

i.e., $\mathfrak{C}_{s}$ is $\mathbf{N}_{x}$ which is in $f^{*^{-1}}\left(\left(t_{f}-\varepsilon, t_{f}+\varepsilon\right)\right)$.

Lemma 3.10 Let $k: \quad \mathbf{Y} \rightarrow Y^{w}$ be defined by $k(x)=\mathbf{N}_{x}$. Then, (i) $k$ is an embedding from $\mathbf{Y}$ into $Y^{w}$; (ii) for all $f$ in $D, f^{*} \circ k=f$; and (iii) $k(\mathbf{Y})$ is dense in $Y^{w}$.

Proof. (i) By the setting, $\mathbf{N}_{x}=\mathbf{N}_{y}$ if $x=y$. Thus $k$ is well-defined and one-one. Let $k^{-1}$ be a function from $k(\mathbf{Y})$ into $\mathbf{Y}$ defined by $k^{-1}(k(x))=x$. To show the continuity of $k$ and $k^{-1}$, for any $F^{*}$ in $\tau$, (a): $\mathrm{x}$ is in

$$
k^{-1}\left(\left[\left(Y^{w}-F^{*}\right)\right] \cap k(\mathbf{Y})\right)
$$

iff (b): $k(\mathrm{x})=\mathrm{N}_{x}$ is in $\left(Y^{w}-F^{*}\right)$. By Cor. 3.5 (i), (b) iff (c): $\mathrm{x}$ is not in $F$. So,

$$
\mathbf{Y}-F=k^{-1}\left[\left(Y^{w}-F^{*}\right) \cap k(\mathbf{Y})\right]
$$

i.e.,

$$
\mathrm{k}(Y-F)=k(\mathbf{Y}) \cap\left(Y^{w}-F^{*}\right) .
$$

So, $k$ and $k^{-1}$ are continuous. (ii) is obvious. (iii) For any $F^{*}$ in $\tau$ such that $Y^{w}-F^{*} \neq \phi$, pick a $\mathfrak{C}$ in $Y^{w}-F^{*}$. By Cor. 3.5 (iv), there is a $T$ in $\mathfrak{C}$ such that $T \subset Y-F$. Pick an $\mathrm{x}$ in $T$, by Cor. 3.5 (i), $k(x)=\mathrm{N}_{\mathrm{x}}$ which is not in $F^{*}$, so $\mathbf{N}_{x}=k(x)$ is in both $k(\mathbf{Y})$ and $\left(Y^{w}-F^{*}\right)$; i.e., $k(\mathbf{Y}) \cap\left(Y^{w}-F^{*}\right) \neq \phi$. Thus, $k(\mathbf{Y})$ is dense in $Y^{w}$.

Let $D^{*}=\left\{f^{*} \mid f \in D\right\}$. Then $D^{*} \subseteq \mathbf{C}^{*}\left(Y^{w}\right)$. Let

$$
\begin{aligned}
& \mathrm{K}^{*}=\left\{\cap_{f^{*} \in H^{*}} f^{*-1}\left(\left[r_{f}-\varepsilon, r_{f}+\varepsilon\right]\right) \mid \cap_{f^{*} \in H^{*}} f^{*-1}\right. \\
& \left.\left(\left[r_{f}-\varepsilon, r_{f}+\varepsilon\right]\right) \neq \phi \text { for any } H^{*} \in\left[D^{*}\right]^{<\omega}, \varepsilon>0\right\}
\end{aligned}
$$

be a closed $C^{*}{ }_{D^{*-f i l t e r}}$ base on $Y^{w}$ and let $\mathcal{E}^{*}$ be the basic closed $C^{*}{ }^{*}$-filter on $Y^{w}$ generated by $K^{*}$. Since $k$ and $k^{-1}$ are one-one, $f^{*} \circ k=f$ for all $f$ in $D$ and $k(\mathbf{Y})$ is dense in $Y^{w}$, so

$$
\begin{aligned}
& \cap_{f^{*} \in H^{*}} f^{*^{-1}}\left(\left(r_{f}-\varepsilon, r_{f}+\varepsilon\right)\right) \cap k(\mathbf{Y}) \\
& =k\left(\cap_{f \in H} f^{-1}\left(\left(r_{f}-\varepsilon, r_{f}+\varepsilon\right)\right)\right)
\end{aligned}
$$

for any $H^{*} \in\left[D^{*}\right]^{<\omega}, H=\left\{f \mid f^{*} \in H^{*}\right\} \quad$ (or any $H \in[D]^{<\omega}, H^{*}=\left\{f^{*} \mid f \cup H\right\}$ and all $\varepsilon>0$. Thus,

$$
\cap_{f^{*} \in H^{*}} f^{*-1}\left(\left(r_{f}-\varepsilon, r_{f}+\varepsilon\right)\right) \neq \phi
$$

iff

$$
\cap_{f \in H} f^{-1}\left(\left(r_{f}-\varepsilon, r_{f}+\varepsilon\right)\right) \neq \phi
$$

and

$$
\cap_{f^{*} \in H^{*}} f^{*-1}\left(\left[r_{f}-\varepsilon, r_{f}+\varepsilon\right]\right) \neq \phi
$$

iff

$$
\cap_{f \in H} f^{-1}\left(\left[r_{f}-\varepsilon, r_{f}+\varepsilon\right]\right) \neq \phi
$$

for any $H^{*} \in\left[D^{*}\right]^{<\omega}, H=\left\{f \mid f^{*} \in H^{*}\right\}$ (or any $H \in[D]^{<\omega}, H^{*}=\left\{f^{*} \mid f \in H\right\}$ and all $\varepsilon>0$. Therefore, if the $\mathrm{K}^{*}$ or $\mathcal{E}^{*}$ defined as above is well-defined, so is $\mathrm{K}$ or $\mathcal{E}$ defined as in Section 2 well-defined and vice versa. If $\mathrm{K}^{*}$ or $\mathcal{E}^{*}$ is given, then $\mathrm{K}$ or $\mathcal{E}$ is called the closed $C^{*}{ }{ }^{-}$-filter base or the basic closed $C^{*}{ }^{*}$-filter on $\mathrm{Y}$ induced by $\mathrm{K}^{*}$ or $\mathcal{E}^{*}$ and vice versa.

Lemma 3.11 Let $\mathcal{E}$ be a basic closed $\boldsymbol{C}^{*}{ }^{*}$-filter on $\mathbf{Y}$ defined as in Section 2. If $\mathcal{E}$ converges to a point $x$ in $\mathbf{Y}$, then $(i) r_{f}=f(x)$ for all $f$ in $D$; i.e. $\mathcal{E}=\mathcal{E}_{x}$, (ii) $\bigvee_{x}$ is an open nhood base at $x$ in $\mathbf{Y}$ and (iii)

$$
\begin{aligned}
& \mathrm{V}_{k(x)}^{*}=\left\{\cap_{f^{*} \in H^{*}} f^{*-1}((f(x)-\varepsilon, f(x)+\varepsilon)) \mid\right. \\
& \left.H^{*} \in\left[D^{*}\right]^{<\omega}, H=\left\{f \mid f^{*} \in H^{*}\right\}, \varepsilon>0\right\}
\end{aligned}
$$

is an open nhood base at $k(x)$ in $Y^{w}$.

Proof. If $\mathcal{E}$ converges to $x$ in $\mathbf{Y}$, (i): for each $f \in D$,

$$
x \in f^{-1}\left(\left[r_{f}-\varepsilon, r_{f}+\varepsilon\right]\right) \in \mathrm{K} \subset \mathfrak{C}
$$

for all $\varepsilon>0$, thus $f(x)=r_{f}$; i.e., $\mathcal{E}=\mathcal{E}_{x}$. (ii): Since $\mathcal{E}$ converges to $x$ in $\mathbf{Y}$, for any open nhood $U$ of $x$, there is

$$
E=\cap_{f \in H} f^{-1}([f(x)-\delta, f(x)+\delta]) \in \mathrm{K}_{x}
$$

which is contained in $\mathcal{E}_{x}=\mathcal{E}$ for some $H \in[D]^{<\omega}, \delta>0$ such that $\in E \subset U$. Since $x$ is in

$$
\mathbf{S}=\cap_{f \in H} f^{-1}((f(x)-\delta, f(x)+\delta)) \subset E \subset U
$$

and $\mathbf{S}$ is in $\mathrm{V}_{x}$, thus $\mathrm{V}_{x}$ is an open nhood base at $x$; (iii): For any $F^{*}$ in $\tau$ such that $\mathrm{N}_{\mathrm{x}}$ is not in $F^{*}$, by Cor. 3.5 (i), $x$ is not in $F$, and by (ii) of Lemma 3.11 above, $x$ is in

$$
\mathrm{O}=\cap_{f \in H} f^{-1}([f(x)-\delta, f(x)+\delta]) \subset Y-F
$$


for some $H \in[D]^{<\omega}, \delta>0$. Since

$$
x \in P=\cap_{f \in H} f^{-1}([f(x)-\delta / 2, f(x)+\delta / 2]) \in \mathrm{N}_{\mathrm{x}},
$$

Cor. 3.5 (i), Lemmas 3.6 (ii) and 3.8 (i) imply that

$$
\begin{aligned}
& \mathrm{N}_{\mathrm{x}} \in P^{*} \subset \cap_{f^{*} \in H^{*}} f^{*^{-1}}((f(x)-\delta, f(x)+\delta)) \\
& =T \in \mathrm{V}_{\mathrm{k}(\mathrm{x})}^{*},
\end{aligned}
$$

where $H^{*}=\left\{f^{*} \mid f \in H\right\}$. We claim that $T \subset Y^{w}-F^{*}$ : For any $\mathfrak{C}_{s}$ in $T$, if $f *\left(\mathfrak{C}_{s}\right)=s_{f}$ for all $f$ in $D$, then $s_{f}$ is in $\mathbf{I}_{\mathrm{f}}=(f(x)-\delta, f(x)+\delta)$ for all $f$ in $H$. Pick a $\rho>0$ such that $\left[s_{f}-\rho, s_{f}+\rho\right] \subset \mathbf{I}_{f}$ for all $f$ in $H$, then

$$
L=\cap_{f \in H} f^{-1}\left(\left[s_{f}-\rho, s_{f}+\rho\right]\right) \subset \mathrm{O} \subset Y-F
$$

and $L \in \mathrm{K}_{\mathrm{s}} \subset \mathfrak{C}_{\mathrm{s}}$; i.e. $\mathfrak{C}_{\mathrm{s}} \in Y^{w}-F^{*}$. So

$$
k(x) \in T \subset Y^{w}-F^{*} .
$$

Thus $\mathrm{V}_{k(x)}^{*}$ is an open nhood base at $k(x)$.

Lemma 3.12 Let $\mathcal{E}$ be a basic $C^{*}{ }^{*}$-filter on $\mathbf{Y}$ defined as in Section 2. If $\mathcal{E}$ does not converge in $\mathbf{Y}$,

$$
\begin{aligned}
& V_{\varepsilon}^{*}=\left\{\cap f^{*} \in H^{*}\right. \\
& \left.H^{*} \in\left[D^{*-1}\right]^{<\omega}, \varepsilon>0\right\}
\end{aligned}
$$

is an open nhood base at $\mathcal{E}$ in $Y^{w}$.

Proof. If $\mathcal{E}$ does not converge in $\mathbf{Y}$, then $\mathcal{E}$ is in $Y^{w}$. Since $f^{*}(\mathcal{E})=r_{f}$ for all $f^{*} \in D^{*}$,

$$
\mathcal{\varepsilon} \in \cap_{f^{*} \in H^{*}} f^{*^{-1}}\left(\left(r_{f}-\varepsilon, r_{f}+\varepsilon\right)\right)
$$

for any $H^{*} \in\left[D^{*}\right]^{<\varepsilon}, \varepsilon>0$. For any $F^{*} \in \tau$ such that $\mathcal{\varepsilon} \in Y^{w}-F^{*}$, by Cor. 3.5 (iv) there exists a

$$
\mathbf{E}=\cap_{f \in H} f^{-1}\left(\left[r_{f}-\delta, r_{f}+\delta\right]\right) \in \mathrm{K} \subset \mathcal{E}
$$

for some $H \in[D]^{<\omega}, \delta>0$ such that $\mathbf{E} \subset \mathbf{Y}-\mathbf{F}$. For $H^{*}=\left\{f^{*} \mid f \in H\right\}$, let

$$
U=\cap_{f^{*} \in H^{*}} f^{*^{-1}}\left(\left(r_{f}-\delta, r_{f}+\delta\right)\right),
$$

then $\mathcal{E} \in U \in \mathrm{V}^{*}$. We claim that $U \subset Y^{w}-F^{*}$. For any $\mathcal{E}_{t}$ in $U$, let $f^{*}\left(\mathcal{E}_{t}\right)=t_{f}$ for each $f^{*}$ in $H^{*}$. Then for each $f$ in $H, t_{f}$ is in

$$
\left(r_{f}-\delta, r_{f}+\delta\right) \text { and } f^{-1}\left(\left[t_{f}-\gamma, t_{f}+\gamma\right]\right) \in \mathcal{E}_{\mathrm{t}}
$$

for all $\gamma>0$. Pick a $\sigma>0$ such that

$$
\left[t_{f}-\sigma, t_{f}+\sigma\right] \subset\left[r_{f}-\delta, r_{f}+\delta\right]
$$

for each $f$ in $H$, then

$$
L=\cap_{f \in H} f^{-1}\left(\left[t_{f}-\sigma, t_{f}+\sigma\right]\right) \subset E \subset Y-F .
$$

Since $L \in \mathrm{K}_{t} \subset \mathcal{E}_{t}$, so $\mathcal{E}_{t} \in Y^{w}-F^{*}$. Hence $\mathcal{E}$ is in
$U \subset Y^{w}-F^{*}$. Thus, $\mathrm{V}^{*} \varepsilon$ is an open nhood base at $\mathcal{E}$.

Proposition 3.13 For any basic closed $C^{*}{ }_{D^{*}-\text { filter }} \mathcal{E}^{*}$ on $Y^{w}, \mathcal{E}^{*}$ converges in $Y^{w}$.

Proof. For given $\mathcal{E}^{*}$, let $\mathrm{K}$ and $\mathcal{\varepsilon}$ be the closed $C^{*}{ }^{*}$-filter base and the basic closed $C^{*}{ }^{*}$-filter on $\mathbf{Y}$ induced by $\mathcal{E}^{*}$. Case 1: If $\mathcal{E}$ converges to an $x$ in $\mathbf{Y}$, then $r_{f}$ is $f(x)$ for all $f$ in $D$. For any

$$
U=\cap_{f^{*} \in H^{*}} f^{*^{-1}}\left(\left(r_{f}-\delta, r_{f}+\delta\right)\right)
$$

in $\mathrm{V}^{*}{ }_{k(x)}$, let

$$
E=\cap_{f^{*} \in I^{*}} f^{*-1}\left(\left[r_{f}-\delta / 2, r_{f}+\delta / 2\right]\right),
$$

where $I^{*} \in\left[D^{*}\right]^{<\omega}$. Then $E \in \mathrm{K}^{*} \subset \mathcal{E}^{*}$ and $E \subset U$. Thus, $\mathcal{E}^{*}$ converges to $k(x)=\mathbf{N}_{\mathrm{x}}$ in $Y^{w}$. Case 2: If $\mathcal{E}$ does not converge in $\mathbf{Y}$, then $\mathcal{E}$ is in $Y^{w}$. For any

$$
U=\cap_{f^{*} \in I^{*}} f^{*^{-1}}\left(\left(r_{f}-\delta, r_{f}+\delta\right)\right)
$$

in $\mathrm{V}^{*} \varepsilon$, let

$$
E=\cap_{f^{*} \in I^{*}} f^{*^{-1}}\left(\left[r_{f}-\delta / 2, r_{f}+\delta / 2\right]\right),
$$

then $E \in \mathrm{K}^{*} \subset \mathcal{E}^{*}$ and $E \subset U$. Thus, $\mathcal{E}^{*}$ converges to $\mathcal{E}$ in $Y^{w}$.

Theorem $3.14\left(Y^{w}, k\right)$ is a compactification of $\mathbf{Y}$.

Proof. First, we show that $Y^{w}$ is compact. Let $G$ be a sub-collection of $\tau$ with the finite intersection property. Let

$$
\mathbf{L}=\left\{\cap_{E^{*} \in H} E^{*} \mid H \in[G]^{<\omega}\right\},
$$

then $\mathbf{L}$ is a filter base on $Y^{w}$. Let $\mathcal{F}$ be a closed ultrafilter on $Y^{w}$ such that $\mathbf{L}$ is contained in $\mathcal{F}$. By Cor. 2.3, there is a unique basic closed $C^{*} D^{*}$-filter $\mathcal{E}^{*}$ on $Y^{w}$ such that $\mathcal{E}^{*}$ is contained in $\mathcal{F}$. By Prop. 3.13, $\mathcal{E}^{*}$ converges to an $\mathcal{E}_{\mathrm{o}}$ in $Y^{w}$. This implies that $\mathcal{F}$ converges to $\mathcal{E}_{\mathrm{o}}$ too. Hence, $\mathcal{E}_{\mathrm{o}}$ is in $\boldsymbol{F}$ for all $\boldsymbol{F}$ in $\mathcal{F}$; i.e., $\mathcal{E}_{\mathrm{o}} \in \cap\left\{E^{*} \mid E^{*} \in G\right\}$. Thm. 17.4 in [1, p.118], $Y^{w}$ is compact. Thus, by Lemma 3.10 (i) and (iii), $\left(Y^{w}, k\right)$ is a compactification of $\mathbf{Y}$.

\section{The Hausdorff Compactification $\left(X^{w}, k\right)$ of $X$ Induced by a Subset $D$ of $C^{*}(X)$}

Let $\mathbf{X}$ be a Tychonoff space and let $D$ be a subset of $\mathbf{C}^{*}(\mathbf{X})$ such that $D$ separates points of $\mathbf{X}$ and the topology on $\mathbf{X}$ is the weak topology induced by $D$. It is clear that $D$ contains a non-constant function. For each $x$ in $\mathbf{X}$, since $\mathrm{V}_{x}$ is an open nhood base at $\mathrm{x}$, it is clear that $\mathcal{E}_{x}$ converges to x. Let $X^{w}=X_{E} \cup X_{F}$, where $X_{E}=\left\{\boldsymbol{\varepsilon}_{x}\right.$ $\mid x \in \mathbf{X}\}$ and $X_{E}=\left\{\mathcal{E} \mid \mathcal{E}\right.$ is a basic closed $C^{*} D^{\text {-filter that }}$ does not converge in $\mathbf{X}$ \}. Similar to what we have done in Section 3, we can get the similar definitions, lemmas, propositions and a theorem in the following:

(4.15.4) (See Def. 3.4) For a nonempty closed set $F$ in $\mathbf{X}, F^{*}=\left\{\mathcal{E} \in X^{w} \mid F \cap T \neq \phi\right.$ for all $T$ in $\left.\mathcal{E}\right\}$. 
(4.15.5) (See Cor. 3.5) For a nonempty closed set $\mathbf{F}$ in $\mathbf{X}$, (i) $x$ is in $\mathbf{F}$ if $\mathcal{E}_{x}$ is in $\mathbf{F}^{*}$; (ii) $\mathbf{F}$ is $\mathbf{X}$ if $\mathbf{F}^{*}=X^{w}$; (iii) for each $\mathcal{E}$ in $X^{w}, \boldsymbol{F}$ is in $\mathcal{E}$ implying $\mathcal{E}$ is in $\mathbf{F}^{*}$; (iv) $\mathcal{E}$ $\in X^{w}-F^{*} \Leftrightarrow$ there is a $S$ in $\mathcal{E}$ such that $S \subset X-F$.

Proof. (i) $(\Leftarrow)$ If $\mathcal{E}_{x}$ is in $F^{*}$, then

$$
\begin{aligned}
& F \cap f^{-1}((f(x)-\varepsilon, f(x)+\varepsilon)) \supset \\
& F \cap f^{-1}([f(x)-\varepsilon / 2, f(x)+\varepsilon / 2]) \neq \phi
\end{aligned}
$$

for all $f$ in $D, \varepsilon>0$. Since $\mathrm{V}_{x}$ is a nhood base at $x$, thus $x$ is a cluster point of $\mathbf{F}$, so $x$ is in $\mathbf{F}$. (i) implying (ii), (iii) and (iv) are obvious.

(4.15.6) (See Lemma 3.6) For any two nonempty sets $E$ and $F$ in $\mathbf{X}$,

(i) $E \subseteq F \Leftrightarrow E^{*} \subseteq F^{*}$;

(ii) $(E \cap F)^{*} \subseteq\left(E^{*} \cap F^{*}\right)$;

(iii) $(E \cup F)^{*}=\left(E^{*} \cup F^{*}\right)$.

(4.15.7) (See Prop. 3.7) $\tau=\left\{\mathbf{F}^{*} \mid \mathbf{F}\right.$ is a nonempty closed set in $\mathbf{X}\}$ is a base for the closed sets of $X^{w}$.

(4.15.7.1) (See the definitions for the topology $\mathfrak{I}$ on $Y^{w}$ and $f^{*}$ for each $f$ in $D$ in Section 3.)

Equip $X^{w}$ with the topology $\mathfrak{I}$ induced by $\tau$. For each $f$ in $D$, define $\mathrm{f}^{*}: X^{w} \rightarrow \mathbf{R}$ by $\mathrm{f}^{*}(\mathcal{E})=r_{f}$ if $f^{-1}\left(\left(r_{f}-\varepsilon, r_{f}+\varepsilon\right)\right) \in \mathcal{E}$ for all $\varepsilon>0$. Then $\mathrm{f}^{*}$ is welldefined and $\mathrm{f}^{*}\left(\mathcal{E}_{x}\right)$ is $f(x)$ for all $f$ in $D$ and all $x$ in $\mathbf{X}$.

(4.15.8) (See Lemma 3.8) For each $f$ in $D$, let $\mathrm{r}$ be in $\mathrm{Cl}(\mathrm{f}(\mathbf{X}))$, then

(i) $\left(f^{-1}([r-\delta, r+\delta])\right) * f^{*-1}((r-\varepsilon, r+\varepsilon))$

and

(ii) $f^{*-1}((r-\varepsilon, r+\varepsilon)) \subseteq\left(f^{-1}([r-\varepsilon, r+\varepsilon])\right) *$

for any $\varepsilon>\delta>0$.

(4.15.9) (See Prop. 3.9) For each $f$ in $D, f^{*}$ is a bounded real continuous function on $X^{w}$.

(4.15.10) (See Lemma 3.10) Let $k: \mathbf{X} \rightarrow X^{w}$ be defined by $k(x)=\mathcal{E}_{\mathrm{x}}$. Then, $(i) k$ is an embedding from $\mathbf{X}$ into $X^{w}$; (ii) $f^{*} \circ k=f$ for all $f$ in $D$; and (iii) $k(\mathbf{X})$ is dense in $X^{w}$.

(4.15.11) (See Lemmas 3.11 and 3.12) For each $\mathcal{E}$ in $X^{w}$, let

$$
\begin{aligned}
& \mathrm{K}=\left\{\cap_{f \in H} f^{-1}\left(\left[r_{f}-\varepsilon, r_{f}+\varepsilon\right]\right) \mid\right. \\
& \cap_{f \in H} f^{-1}\left(\left[r_{f}-\varepsilon, r_{f}+\varepsilon\right]\right) \neq \phi \\
& \text { for a ny } \left.H \in[D]^{<\omega}, \varepsilon>0\right\} \subset \mathcal{E}
\end{aligned}
$$

1) If $\mathcal{E}$ converges to $x$, then $\mathcal{E}$ is $\mathcal{E}_{x}$ and $\mathrm{V}^{*}\left({ }_{k}\right)$ is $=$

$$
\begin{aligned}
& \mathrm{V}^{*} \varepsilon_{x}= \\
& \left\{\cap_{f^{*} \in H^{*}} f^{*-1}((f(x)-\varepsilon, f(x)+\varepsilon)) \mid H^{*} \in\left[D^{*}\right]^{<\omega},\right. \\
& \varepsilon>0\}
\end{aligned}
$$

is an open nhood base at $\mathcal{E}_{x}$. 2) If $\mathcal{E}$ does not converge in $\mathbf{X}$, then $\mathcal{E}$ is in $X^{w}$ and

$$
\begin{aligned}
& \mathrm{V}_{\varepsilon}= \\
& \left\{\cap_{f^{*} \in H^{*}} f^{*-1}\left(\left(r_{f}-\varepsilon, r_{f}+\varepsilon\right)\right) \mid\right. \\
& \cap_{f^{*} \in H^{*}} f^{*-1}\left(\left(r_{f}-\varepsilon, r_{f}+\varepsilon\right)\right) \\
& \left.\neq \phi \text { for any } H^{*} \in\left[D^{*}\right]^{<\omega}, \varepsilon>0\right\}
\end{aligned}
$$

is an open nhood base at $\mathcal{E}$ in $X^{w}$.

(4.15.13) (See Prop. 3.13) Each basic closed $\boldsymbol{C}^{*}{ }^{*-}$ filter $\mathcal{E}^{*}$ on $X^{w}$ converges to $\mathcal{E}$ in $X^{w}$.

(4.15.14) (See Theorem 3.14) $\left(X^{w}, k\right)$ is a compactification of $\mathbf{X}$.

Lemma 4.16 $D^{*}$ separates points of $X^{w}$.

Proof. For $\mathcal{E}_{s}, \mathcal{E}_{t}$ in $X^{w}$, let

$$
\begin{aligned}
& \mathrm{K}_{s}=\left\{\cap_{f \in H} f^{-1}\left(\left[s_{f}-\varepsilon, s_{f}+\varepsilon\right]\right) \mid\right. \\
& \cap_{f \in H} f^{-1}\left(\left[s_{f}-\varepsilon, s_{f}+\varepsilon\right]\right) \\
& \left.\neq \phi \text { for any } H \in[D]^{<\omega}, \varepsilon>0\right\}
\end{aligned}
$$

and similarly for $\mathrm{K}_{t}$. Since $\mathcal{E}_{s}$ is not equal to $\mathcal{E}_{t}, \mathrm{~K}_{s}$ is not equal to $\mathrm{K}_{t}$ and that $D$ has a $\mathrm{g}$ such that $s_{g} \neq t_{g}$ are equivalent, where $g^{-1}\left(\left[s_{g}-\varepsilon, s_{g}+\varepsilon\right]\right) \in \mathrm{K}_{s}$ which is contained in $\mathcal{E}_{s}$ and $g^{-1}\left(\left[s_{g}-\varepsilon, s_{g}+\varepsilon\right]\right) \in \mathrm{K}_{s}$ which is contained in $\mathcal{E}_{t}$ for all $\varepsilon>0$, thus by the definition of $\mathrm{g}^{*}$, $\mathrm{g}^{*}\left(\mathcal{E}_{s}\right)=s_{g} \neq t_{g}=\mathrm{g} *\left(\mathcal{E}_{t}\right)$.

Theorem $4.17\left(X^{w}, k\right)$ is a Hausdorff compactification of $\mathbf{X}$.

Proof. By 4.15.10 (i) and (iii), 4.15.14 and Lemma 4.16, $\left(X^{w}, k\right)$ is a Hausdorff compactification of $\mathbf{X}$.

\section{The Homeomorphism between $\left(X^{w}, k\right)$ and (Z,h)}

Let $(\mathbf{Z}, h)$ be an arbitrary Hausdorff compactification of $\mathbf{X}$, then $\mathbf{X}$ is a Tychonoff space. Let ${ }^{\circ} D$ denote $\mathbf{C}(\mathbf{Z})$ which is the family of real continuous functions on $\mathbf{Z}$, and let $D=\left\{f \mid f={ }^{\circ} f \circ h,{ }^{\circ} f \in{ }^{\circ} D\right\}$. Then $D$ is a subset of $\mathbf{C}^{*}(\mathbf{X})$ such that $D$ separates points of $\mathbf{X}$, the topology on $\mathbf{X}$ is the weak topology induced by $D$ and $D$ contains a non-constant function.

Let $\left(X^{w}, k\right)$ be the Hausdorff compactification of $\mathbf{X}$ obtained by the process in Section 4 and $D$ is defined as above. For each basic closed $C^{*}{ }_{D}$-filter $\mathcal{E}$ in $X^{w}$, let $\mathcal{E}$ be generated by

$$
\begin{aligned}
& \mathrm{K}=\left\{\cap_{f \in H} f^{-1}\left(\left[r_{f}-\varepsilon, r_{f}+\varepsilon\right]\right) \mid \cap_{f \in H} f^{-1}\right. \\
& \left.\left(\left[r_{f}-\varepsilon, r_{f}+\varepsilon\right]\right) \neq \phi \text { for any } H \in[D]^{<\omega}, \varepsilon>0\right\}
\end{aligned}
$$

let ${ }^{\circ} \mathcal{E}$ be the basic closed $C^{*}{ }_{D}$-filter on $\mathbf{Z}$ generated by 


$$
\begin{aligned}
& { }^{\circ} \mathrm{K}=\left\{\cap_{{ }_{f \in} \in^{\circ} H}{ }^{\circ} f^{-1}\left(\left[r_{f}-\varepsilon, r_{f}+\varepsilon\right]\right) \mid \cap_{\circ f \in{ }^{\circ} H}{ }^{\circ} f^{-1}\right. \\
& \left.\left(\left[r_{f}-\varepsilon, r_{f}+\varepsilon\right]\right) \neq \phi \text { for any }{ }^{\circ} H \in\left[{ }^{\circ} D\right]^{<\omega}, \varepsilon>0\right\}
\end{aligned}
$$

and let $h^{-1}$ be the function from $\mathrm{h}(\mathbf{X})$ to $\mathbf{X}$ defined by $h^{-1}(h(x))=x$. Since $h$ and $h^{-1}$ are one-one, $f={ }^{\circ} f$ o $h$ and $\mathrm{h}(\mathbf{X})$ is dense in $\mathbf{Z}$, similar to the arguments in the paragraphs prior to Lemma 3.11, we have that

$$
\cap_{f \in H} f^{-1}\left(\left[r_{f}-\varepsilon, r_{f}+\varepsilon\right]\right) \neq \phi
$$

iff

$$
\cap_{f \in \in^{\circ} H}^{\circ} f^{-1}\left(\left[r_{f}-\varepsilon, r_{f}+\varepsilon\right]\right) \neq \phi
$$

for any

$$
\begin{aligned}
& { }^{\circ} H \in\left[{ }^{\circ} D\right]^{<\omega} \quad \text { (or any } H \in[D]^{<\omega} \text { ), } \\
& H=\left\{\left.f\right|^{\circ} f \in{ }^{\circ} H\right\} \quad \text { (or }{ }^{\circ} H=\left\{{ }^{\circ} f \mid f \in H\right\} \text { ) }
\end{aligned}
$$

and all $\varepsilon>0$. Thus, if $\mathrm{K}$ or $\mathcal{E}$ is well-defined, so is ${ }^{\circ} \mathrm{K}$ or ${ }^{\circ} \mathcal{E}$ and vice versa. If $\mathrm{K}$ or $\mathcal{E}$ is given, ${ }^{\circ} \mathrm{K}$ or ${ }^{\circ} \mathcal{E}$ is called the closed $C^{*_{\circ}}{ }_{D}$-filter base or the basic closed $C^{*_{\circ}}$-filter on $\mathbf{Z}$ induced by $\mathrm{K}$ or $\mathcal{E}$ and vice versa. For any $z$ in $\mathbf{Z}$,

$$
\begin{aligned}
& { }^{\circ} \mathrm{K}_{z}=\left\{\cap_{\circ}{ }_{f \in{ }^{\circ} H}{ }^{\circ} f^{-1}\left(\left[{ }^{\circ} f(z)-\varepsilon,{ }^{\circ} f(z)+\varepsilon\right]\right)\right. \\
& \left.{ }^{\circ} H \in\left[{ }^{\circ} D\right]^{<\omega}, \varepsilon>0\right\}
\end{aligned}
$$

is the closed $C^{*_{\circ}} D^{-}$filter base at $\mathbf{z}$. The closed filter ${ }^{\circ} \mathcal{E}_{\mathrm{z}}$ generated by ${ }^{\circ} \mathrm{K}_{z}$ is the basic closed $C^{*_{\circ}}{ }$-filter at $\mathrm{z}$. Since $\mathbf{Z}$ is compact Hausdorff, each ${ }^{\circ} \mathcal{E}$ on $\mathbf{Z}$ converges to a unique point $z$ in $\mathbf{Z}$. So, we define $\mathrm{T}: X^{w} \rightarrow \mathbf{Z}$ by $\mathrm{T}(\mathcal{E})=z$, where $\mathcal{E}$ is in $X^{w}$ and $\mathrm{z}$ is the unique point in $\mathbf{Z}$ such that the basic closed $C^{*_{\circ}} D$-filter ${ }^{\circ} \mathcal{E}$ on $\mathbf{Z}$ induced by $\mathcal{E}$ converges to it. For $\mathcal{E}_{s}, \mathcal{E}_{t}$ in $X^{w}$, let

$$
\begin{aligned}
& \mathrm{K}_{\mathrm{s}}=\left\{\cap_{f \in H} f^{-1}\left(\left[s_{f}-\varepsilon, s_{f}+\varepsilon\right]\right) \mid \cap_{f \in H} f^{-1}\right. \\
& \left.\left(\left[s_{f}-\varepsilon, s_{f}+\varepsilon\right]\right) \neq \phi \text { for any } H \in[D]^{<\omega}, \varepsilon>0\right\}
\end{aligned}
$$

and similarly for $\mathrm{K}_{t}$ such that $\mathcal{E}_{s}$ and $\mathcal{E}_{t}$ are generated by $\mathrm{K}_{s}$ and $\mathrm{K}_{t}$, respectively. Assume that ${ }^{\circ} \mathcal{E}_{s}$ and ${ }^{\circ} \mathcal{E}_{t}$ converge to $\mathrm{Z}_{s}$ and $z_{t}$ in $\mathbf{Z}$, respectively. Then $\mathcal{E}_{s}$ is not equal to $\mathcal{E}_{t}$, ${ }^{\circ} \mathcal{E}_{s}$ is not equal to ${ }^{\circ} \mathcal{E}_{t}$ and $z_{s}$ is not equal to $z_{t}$ are equivalent. Hence $\mathrm{T}$ is well-defined and one-one. For each $\mathrm{z}$ in $\mathbf{Z}$, let ${ }^{\circ} \mathcal{E}$, be the basic closed $C^{*_{o}}{ }$-filter at $z$, since $\mathbf{Z}$ is compact Hausdorff and

$$
\begin{aligned}
& { }^{\circ} \mathrm{V}_{z}=\left\{\cap^{\circ} f \in^{\circ} H\right. \\
& \left.{ }^{\circ} H \in\left[{ }^{\circ} D\right]^{<\omega}, \varepsilon>0\right\}
\end{aligned}
$$

is an open nhood base at $\mathrm{z}$, thus ${ }^{\circ} \mathcal{E}_{z}$ converges to z. Let $\mathcal{E}_{z}$ be the element in $X^{w}$ induced by ${ }^{\circ} \mathcal{E}_{z}$, then, $\mathrm{T}\left(\mathcal{E}_{z}\right)=z$. Hence, $\mathrm{T}$ is one-one and onto.
Theorem 5.18 ( $\left(X^{w}, k\right)$ is homeomorphic to $(\mathbf{Z}, h)$ under the mapping $\mathrm{T}$ such that $\mathrm{T}(k(x))=h(x)$.

Proof. We show that $\mathrm{T}^{-1}$ is continuous. For each $\mathcal{E}$ in $\mathbf{F}^{*}$ which is in $\tau$, let ${ }^{\circ} \mathcal{E}$ be the basic closed $C^{*_{\circ}} D^{-}$-filter on $\mathbf{Z}$ induced by $\mathcal{E}$. If ${ }^{\circ} \mathcal{E}$ converges to $\mathbf{z}$ in $\mathbf{Z},{ }^{\circ} f(z)=r_{f}$ for each $f$ in $D$ and

$$
\begin{aligned}
& { }^{\circ} \mathrm{K}=\left\{\cap_{\circ}{ }_{f \in \in^{\circ} H}{ }^{\circ} f^{-1}\left(\left[r_{f}-\varepsilon, r_{f}+\varepsilon\right]\right) \mid \cap_{\circ}{ }_{{ }_{f} \in^{\circ} H}{ }^{\circ} f^{-1}\right. \\
& \left.\left(\left[r_{f}-\varepsilon, r_{f}+\varepsilon\right]\right) \neq \phi \text { for any }{ }^{\circ} H \in\left[{ }^{\circ} D\right]^{<\omega}, \varepsilon>0\right\} \subset{ }^{\circ} \mathcal{E}
\end{aligned}
$$

Then (a): $\mathcal{E}$ is in $\mathbf{F}^{*}$ iff (b):

$$
F \cap\left(\cap_{f \in H} f^{-1}\left(\left[r_{f}-\varepsilon, r_{f}+\varepsilon\right]\right)\right) \neq \phi
$$

for any $H \in[D]^{<\omega}, \varepsilon>0$, where

$$
\cap_{f \in H} f^{-1}\left(\left[r_{f}-\varepsilon, r_{f}+\varepsilon\right]\right) \in \mathcal{E} .
$$

Since $h$ is one-one, $f={ }^{\circ} f \circ h$ for all $f$ in $D$, so (b) iff (c):

$$
\begin{aligned}
& h(F) \cap\left[\cap_{{ }_{f \in}^{\circ} H}^{\circ} f^{-1}\left(\left[r_{f}-\varepsilon, r_{f}+\varepsilon\right]\right)\right] \\
& =h\left(F \cap\left[\cap_{f \in H} f^{-1}\left(\left[r_{f}-\varepsilon, r_{f}+\varepsilon\right]\right)\right]\right) \neq \phi
\end{aligned}
$$

for any

$$
\begin{aligned}
& H \in[D]^{<\omega} \quad\left(\text { or }^{\circ} H \in\left[{ }^{\circ} D\right]^{<\omega}\right), \\
& { }^{\circ} H=\left\{{ }^{\circ} f \mid f \in H\right\} \quad\left(\text { or } H=\left\{\left.f\right|^{\circ} f \in{ }^{\circ} H\right\}\right)
\end{aligned}
$$

and any $\varepsilon>0$. Since

$$
{ }^{\circ} f^{-1}\left(\left(r_{f}-\varepsilon, r_{f}+\varepsilon\right)\right) \supset{ }^{\circ} f^{-1}\left(\left[r_{f}-\varepsilon / 2, r_{f}+\varepsilon / 2\right]\right)
$$

for any ${ }^{\circ} \mathrm{f}$ in ${ }^{\circ} D, \varepsilon>0$, (c) iff (d):

$$
h(F) \cap\left[\cap_{{ }_{f \in \in^{\circ} H}} f^{-1}\left(\left(r_{f}-\varepsilon, r_{f}+\varepsilon\right)\right)\right] \neq \phi
$$

for any ${ }^{\circ} H \in\left[{ }^{\circ} D\right]^{<\omega}, \varepsilon>0$. Since

$$
\cap_{\circ \in \in^{\circ} H}^{\circ} f^{-1}\left(\left(r_{f}-\varepsilon, r_{f}+\varepsilon\right)\right)
$$

is an arbitrary basic open nhood of $z$ in $\mathbf{Z}$. So, (d) iff $z$ is in $\mathrm{Cl}_{\mathrm{z}}(\mathrm{h}(\mathbf{F}))$; i.e., $\mathcal{E}$ is in $\mathbf{F}^{*}$ if $\mathrm{T}(\mathcal{E})$ is equal to $z$ which belongs to $\mathrm{Cl}_{\mathrm{z}}(\mathrm{h}(\mathbf{F}))$. Hence, $\mathrm{T}\left(\mathbf{F}^{*}\right)=\mathrm{Cl}_{\mathrm{z}}(\mathrm{h}(\mathbf{F}))$ is closed in $\mathbf{Z}$ for all $\mathbf{F}^{*}$ in $\tau$. Thus, $\mathrm{T}^{-1}$ is continuous. Since $\mathrm{T}$ is one-one, onto and both $\mathbf{Z}$ and $X^{w}$ are compact Hausdorff, by Theorem 17.14 in [1, p.123], T is a homeomorphism. Finally, from the definitions of $k$ and $h$, it is clear that $\mathrm{T}(k(x))=h(x)$ for all $x$ in $\mathbf{X}$.

Corollary 5.19 Let $(\beta \mathbf{X}, h)$ be the Stone-Čech compactification of a Tychonoff space $\mathbf{X}$,

$$
D=\left\{f \mid f={ }^{\circ} f \circ h,{ }^{\circ} f \in C(\beta X)\right\}
$$

and $\mathrm{T}_{\beta}: X^{w} \rightarrow \beta X$ is defined similarly to $\mathrm{T}$ as above. Then $(\beta \mathbf{X}, h)$ is homeomorphic to $\left(X^{w}, k\right)$ such that $\mathrm{T}_{\beta}(k(\mathrm{x}))=h(\mathrm{x})$. 
Corollary 5.20 Let $(\gamma \mathbf{X}, h)$ be the Wallman compactification of a normal $\mathbf{T}_{1}$-space $\mathbf{X}$,

$$
D=\left\{f \mid f={ }^{\circ} f \circ h,{ }^{\circ} f \in C(\gamma X)\right\}
$$

and $\mathrm{T}_{\gamma}: X^{w} \rightarrow \gamma X$ is defined similarly to $\mathrm{T}$ as above. Then $(\gamma \mathbf{X}, h)$ is homeomorphic to $\left(X^{w}, k\right)$ such that $\mathrm{T}_{\gamma}(k(\mathrm{x}))=h(\mathrm{x})$.

\section{REFERENCES}

[1] S. Willard, "General Topology," Addison-Wesley, Reading, 1970.

[2] H. J. Wu and W. H. Wu, "An Arbitrary Hausdorff Compactification of a Tychonoff Space X Obtained from a $C^{*}{ }$-Base by a Modified Wallman Method," Topology and its Applications, Vol. 155, No. 11, 2008, pp. 11631168. doi:10.1016/j.topol.2007.05.021 